\title{
Classification of two-orbit varieties
}

\author{
Stéphanie Cupit-Foutou
}

\begin{abstract}
We obtain the classification of two-orbit varieties, i.e. the normal complete complex algebraic varieties on which a reductive complex algebraic group acts with two orbits. We prove also Luna's conjecture saying that these varieties are spherical, i.e. admit a dense orbit of a Borel subgroup.
\end{abstract}

Mathematics Subject Classification (2000). 14M17, 20 G05.

Keywords. Two-orbit variety, spherical variety, homogeneous embedding theory, reductive algebraic group.

\section{Introduction}

Our base field is the field $\mathbb{C}$ of complex numbers. We study normal complete algebraic varieties $X$ endowed with an action of a connected reductive algebraic group $G$ admitting a dense orbit $G \cdot x$. The stabilizer $H$ of a point of the dense orbit will be called generic stabilizer. Clearly, $H$ is parabolic if and only if $X=G \cdot x$.

If $H$ is connected, Borel showed in [4] that the homogeneous space $G / H$ has at most two ends, i.e. the complement $X \backslash G \cdot x$ has at most two connected components. When $X \backslash G \cdot x$ is disconnected, the generic stabilizer contains a maximal unipotent subgroup $U$ of $G$. This property results from the connectedness (proved in $[3,11]$ ) of the $U$-fixed point set of $X$. In this situation, the varieties $X$ share other peculiarities which were clarified by Ahiezer in [1] where he studied and classified these objects.

This article deals with the situation where $X \backslash G \cdot x$ is a single orbit and in particular connected. Actually, this case appears as the most natural and "simplest" one to consider after the case of projective homogeneous varieties (Grassmanianns, flag varieties, etc.). We call such varieties two-orbit $G$-varieties or simply two-orbit varieties when no confusion is possible.

Some of these varieties have been studied before. In [2] Ahiezer gave a classification of the pairs $(G, H)$ such that $G \cdot x=G / H$ admits a compactification $X$ by one homogeneous divisor. This list was independently obtained by Huckleberry and Snow [12] in the more general context of kählerian varieties. Later, Brion gave 
in $[7,8]$ a purely algebraic approach to these results using the general theory of embeddings developed by Luna and Vust (see [14]). Finally, Feldmüller classified in [10] all pairs $(G, H)$ giving raise to two-orbit varieties whose closed orbit is of codimension 2 .

The aim of this paper is to give the complete classification of two-orbit varieties and to prove Luna's conjecture saying that two-orbit varieties are spherical, i.e. admit a dense orbit of a Borel subgroup $B$ of $G$. In particular, two-orbit varieties contain only finitely many $B$-orbits. We refer the reader to $[14,6]$ for an introduction to spherical varieties.

Acknowledgements. I would like to thank my adviser Peter Littelmann for his constant support. I am also grateful to Michel Brion for his advice and remarks about my $\mathrm{PhD}$ thesis in which I obtained the results presented in this paper (see $[9])$.

Note added in proof. After this article has been submitted I learned from Alexander Smirnov that he had found a similar classification.

\section{Notation and main results}

Let $G$ be a connected reductive algebraic group. We fix a Borel subgroup $B$ and a maximal torus $T$ in $B$. Let $\Phi$ be the root system of $G$, we denote $\Phi^{+} \subset \Phi$ the set of positive roots relative to $B$ and $\Delta \subset \Phi^{+}$the set of simple roots corresponding to $B$. In case of a simple group $G$, we enumerate the simple roots as in [5] and when it is more convenient, we use the short notation of [loc. cit.] for the description of positive roots. For example, if $G$ is of type $F_{4}, 1111$ stands for the root $\alpha_{1}+\alpha_{2}+\alpha_{3}+\alpha_{4}$. Given some roots $\gamma_{1}, \ldots, \gamma_{r}$, we put $\left\langle\gamma_{1}, \ldots, \gamma_{r}\right\rangle=\Sigma_{i} \mathbb{Q} \gamma_{i} \cap \Phi$.

As usual, we denote the Lie algebra of any connected algebraic subgroup $H$ of $G$ by the corresponding small gothic letter $\mathfrak{h}$. In particular, we have $\mathfrak{g}=$ Lie $G$ and $\mathfrak{t}=$ Lie $T$. For a root $\alpha$ of $G$, we have the corresponding root group $U_{\alpha}$ with Lie algebra $\mathfrak{g}_{\alpha}$. We let $Y_{\alpha}$ be any non-zero element of $\mathfrak{g}_{\alpha}$ so that $\mathbb{C} Y_{\alpha}=\mathfrak{g}_{\alpha}$.

Throughout this article, $X$ will be a two-orbit $G$-variety. If $G \cdot x$ is the dense orbit and $G \cdot y$ is the closed one, we have: $X=G \cdot x \cup G \cdot y=\mathrm{Cl}(G \cdot x)$ (the closure of $G \cdot x)$. An embedding of an homogeneous space $G / H$ is a normal $G$-variety $X^{\prime}$ together with a $G$-equivariant open embedding $G / H \rightarrow X^{\prime}$. Thus, $X$ together with the inclusion $G \cdot x \subset X$ is a complete embedding of $G / H$ with two orbits where $H=G_{x}$.

The following proposition collects some important properties of complete varieties with two orbits.

Proposition 2.1. Let $G$ be a connected reductive algebraic group.

(i) A two-orbit G-variety $X$ is projective. More precisely, $X$ is $G$-equivariantly 
isomorphic to a closed subvariety of $\mathbb{P}(V)$ where $V$ is a rational representation of $G$.

(ii) Let $X^{\prime}$ be a projective $G$-variety with two orbits. Then the normalization $\eta: \widetilde{X}^{\prime} \rightarrow X^{\prime}$ is bijective. In particular, $\widetilde{X}^{\prime}$ is a two-orbit $G$-variety.

(iii) Let $H \subset G$ be a closed subgroup and $H^{\circ}$ its identity component. Then there is a natural bijective correspondence between the two-orbit varieties with generic stabilizer $H$ and those with generic stabilizer $H^{\circ}$.

(iv) The radical $R(G)$ of $G$ acts trivially on every two-orbit $G$-variety.

Proof. (i) By Sumihiro [16, Theorem 1] the closed orbit has a quasi-projective $G$-stable neighbourhood which has to be all of $X$.

(ii) From the birationality of $\eta$ it follows that $\tilde{X}^{\prime}$ has a dense $G$-orbit isomorphic to the dense orbit $G \cdot x^{\prime}$ of $X^{\prime}$. Since $\eta$ is finite and the stabilizer of the closed orbit $G \cdot y^{\prime}$ in $X^{\prime}$ is connected, the inverse image $\eta^{-1}\left(G \cdot y^{\prime}\right)$ is a finite union of closed orbits which are mapped isomorphically onto $G \cdot y^{\prime}$. If there were more than one then, using again the connectedness of the $U$-fixed point set, $U$ would have a fixed point in $G \cdot \widetilde{x}^{\prime}$ hence a fixed point $z^{\prime}$ in $G \cdot x^{\prime}$. Since $X^{\prime}$ is projective it follows that the closure $\mathrm{Cl}\left(T \cdot z^{\prime}\right)$ has at least two $T$-fixed points ([13, Theorem 25.2]) and so $B=T U$ would fix at least two points in $X^{\prime}$ which is impossible.

(iii) Clearly, if $X$ is a complete two-orbit embedding of $G / H^{\circ}, G / H$ inherits a complete two-orbit embedding given by $X / \Gamma$ where $\Gamma$ is the finite group $H / H^{\circ}$. Conversely, if $Z$ is a two-orbit variety with dense orbit $G \cdot z$ and stabilizer $G_{z}=H$ then we consider the normalization $X$ of $Z$ in the field extension $\mathbb{C}\left(G / H^{\circ}\right) / \mathbb{C}(G / H)$. It follows that $X$ has a dense orbit $G \cdot x$ with stabilizer $G_{x}=H^{\circ}$ and that $Z$ is the quotient by $\Gamma$. The same arguments as in (ii) show that $X$ is a two-orbit variety.

(iv) If the action of $R(G)$ on a two-orbit $G$-variety is non-trivial then the fixed point set $X^{R(G)}$ will have at least two connected components [3]. But clearly, $X^{R(G)}$ is equal to the closed $G$-orbit which is connected.

As a consequence, we will assume from now on that $G$ is semisimple. Moreover, part (iii) of the proposition above allows us to restrict our classification to the situation where the generic stabilizer is connected.

Before stating the main results, we have to introduce another construction, called parabolic induction. Let us give first an example.

Let $G$ be a simple group of rank 2 and $\lambda$ a dominant weight such that $\left(\lambda, \alpha_{1}^{\vee}\right)>$ 1 and $\left(\lambda, \alpha_{2}^{\vee}\right)=0$, where $(\cdot, \cdot)$ is the Killing form of $\mathfrak{g}$ and $\alpha_{i}^{\vee}$ the coroot associated to the simple root $\alpha_{i}$. We consider the irreducible $G$-module $V(\lambda)$ associated to $\lambda$, a weight vector $v_{\lambda-\alpha_{1}} \in V(\lambda)$ of weight $\lambda-\alpha_{1}$ and we denote by $\left[v_{\lambda-\alpha_{1}}\right]$ the corresponding element in the projective space $\mathbb{P}(V(\lambda))$. Let $P \supset B$ be the parabolic subgroup associated to $\alpha_{1}$. Then $\operatorname{Cl}\left(P \cdot\left[v_{\lambda-\alpha_{1}}\right]\right) \subset \mathbb{P}(V(\lambda))$ is a $P$ variety with two $P$-orbits, namely $P \cdot\left[v_{\lambda}\right]$ and $P \cdot\left[v_{\lambda-\alpha_{1}}\right]$. Note that the strict 
inequality $\left(\lambda, \alpha_{1}^{\vee}\right)>1$ guarantees that $\left[v_{\lambda}\right]$ and $\left[v_{\lambda-\alpha_{1}}\right]$ are not in the same $P$ orbit. And in turn, $\mathrm{Cl}\left(G \cdot\left[v_{\lambda-\alpha_{1}}\right]\right)=G \cdot \mathrm{Cl}\left(P \cdot\left[v_{\lambda-\alpha_{1}}\right]\right)$ and $G \times{ }_{P} \mathrm{Cl}\left(P \cdot\left[v_{\lambda-\alpha_{1}}\right]\right)$ are $G$-varieties with two $G$-orbits. We have an obvious $G$-equivariant morphism $G \times{ }_{P} \mathrm{Cl}\left(P \cdot\left[v_{\lambda-\alpha_{1}}\right]\right) \rightarrow \mathrm{Cl}\left(G \cdot\left[v_{\lambda-\alpha_{1}}\right]\right)$. This morphism is an isomorphism on the open $G$-orbits and corresponds to the projection $G / B \rightarrow G / Q$ on the closed $G$-orbits where $Q \supset B$ is the parabolic subgroup associated to $\alpha_{2}$.

This example motivates the following notion inspired by Luna's work [15] (see also [2]). From now on, a two-orbit $P$-variety, for $P$ a parabolic subgroup of $G$, is a normal complete $P$-variety with two $P$-orbits on which the unipotent radical of $P$ acts trivially.

Definition. A two-orbit $G$-variety $X$ is said to be obtained by parabolic induction from a pair $(P, Y)$ if $P \subsetneq G$ is a parabolic subgroup and $Y$ a two-orbit $P$-variety such that

(i) the (full) radical of $P$ acts trivially on $Y$;

(ii) there exists a $P$-equivariant injective morphism $\varphi: Y \rightarrow X$ inducing a birational morphism $G \times{ }_{P} Y \rightarrow X$.

A two-orbit variety is cuspidal if it can not be obtained by parabolic induction.

Remark. If $X$ is obtained from $(P, Y)$ by parabolic induction then it is obvious that $X$ is spherical if and only if the two-orbit $L$-variety $Y$ is spherical where $L=P / U_{P}, U_{P}$ being hte unipotent radical of $P$.

Theorem 2.2. (i) As an embedding of its dense G-orbit, a two-orbit G-variety obtained by parabolic induction from a pair $(P, Y)$ is completely determined (up to isomorphism) by its closed $G$-orbit and by the P-variety $Y$.

(ii) Each (non cuspidal) two-orbit variety is obtained by parabolic induction from a unique pair $(P, Z)$ such that $Z$ is cuspidal.

This result allows to restrict the classification to cuspidal two-orbit varieties. In the sections 5 and 6 we determine the possible pairs $(G, H)$ where $G$ is semisimple and $H \subset G$ is the generic stabilizer of a two-orbit $G$-variety (see the first two columns of the table). Then we use the theory of Luna-Vust to show that for these pairs the homogeneous space $G / H$ admits a unique complete two-orbit embedding (see the last column of the table). Thus we get our main result.

Theorem 2.3. The cuspidal two-orbit varieties are obtained as embeddings of the homogeneous spaces $G / H$ with $(G, \mathfrak{h})$ listed in the first two columns of the table. In particular, $G$ is simple or equal to $S L_{2} \times S L_{2}$, in this situation. Moreover, each such $G / H$ has exactly one complete two-orbit embedding given in the last column of the table.

Corollary 2.4. Two-orbit varieties are spherical. 


\section{Cuspidal two-orbit varieties}

$S L_{3}$

$S L_{n+1}$

$\mathrm{SO}_{5}$

or $G_{2}$,

$\operatorname{Spin}_{7}$,

$\mathrm{SO}_{2 n+1}$,

$S O_{2 n+1}$,

$\mathrm{SO}_{2 n+1}$

or $S p_{2 n}$,

$S p_{6}$,

$$
\operatorname{Lie}\left(\operatorname{ker}\left(\alpha_{1}-\alpha_{2}\right)\right) \oplus \mathbb{C}\left(Y_{\alpha_{1}}+Y_{\alpha_{2}}\right) \oplus \bigoplus_{\alpha \in \Phi^{+} \backslash\left\{\alpha_{1}, \alpha_{2}\right\}} \mathfrak{g}_{\alpha},
$$

$\mathfrak{g l}_{n}$

$$
\operatorname{Lie}\left(\operatorname{ker}\left(\alpha_{1}-\alpha_{2}\right)\right) \oplus \mathbb{C}\left(Y_{\alpha_{1}}+Y_{\alpha_{2}}\right) \oplus \bigoplus_{\alpha \in \Phi^{+} \backslash\left\{\alpha_{1}, \alpha_{2}\right\}} \mathfrak{g}_{\alpha},
$$

$\mathfrak{g}_{2}$,

$\mathfrak{s o}_{2 n}$,

$$
\mathfrak{t} \oplus \bigoplus_{\alpha \in \Psi} \mathfrak{g}_{\alpha} \text { with } \Psi=\left\langle \pm \alpha_{1}, \ldots, \pm \alpha_{n-1}, \alpha_{n-1}+2 \alpha_{n}\right\rangle
$$

$$
\mathfrak{t} \oplus \bigoplus_{\alpha \in \Psi} \mathfrak{g}_{\alpha} \text { with } \Psi=\left\langle \pm \alpha_{2}, \ldots, \pm \alpha_{n-1}, \alpha_{n}, \varepsilon_{1}+\varepsilon_{n}\right\rangle
$$

$$
\mathfrak{s l}_{2} \oplus \mathbb{C} \oplus \mathbb{C}\left(Y_{-\alpha_{2}}+Y_{\varepsilon_{1}+\varepsilon_{2}}\right) \oplus
$$
$\alpha \in \Phi^{+} \backslash\left\{\alpha_{2}, \varepsilon_{1}+\varepsilon_{2}, \varepsilon_{1}-\varepsilon_{3}, 2 \varepsilon_{2}\right\}$

with $\mathfrak{t} \cap \mathfrak{s l}_{2} \oplus \mathbb{C}=\operatorname{Lie}\left(\operatorname{ker}\left(\varepsilon_{1}+2 \varepsilon_{2}-\varepsilon_{3}\right)\right)$,
$\left\{(A, z) \in \mathfrak{s l}_{3} \times \mathbb{P}^{2}:\right.$

$A$ nilpotent and $A z=0\}$

$\mathbb{P}^{n} \times \mathbb{P}^{n^{*}}$

$\mathrm{Cl}\left(G \cdot\left[v_{\lambda}+v_{s_{1} s_{2}(\lambda)}\right]\right) \subset \mathbb{P}(V(\lambda))$

with $\lambda=\omega_{1}+\omega_{2}$

$\mathrm{Q}(8)$ action induced by $\mathrm{SO}_{8}$ 's

$\mathrm{Q}(2 n+1)$

$\left\{(\ell, P) \in \mathbb{P}^{2 n+1} \times \mathrm{Gr}(2 n+1 ; n):\right.$

$\ell \subset P$ and $P$ totally isotropic $\}$

$G / P_{1} \times G / P_{n}$

$\mathfrak{g}_{\alpha} \quad\left\{z=\left[\sum_{i, j} v_{i} \wedge v_{j}\right] \in \mathbb{P}\left(\wedge^{2} \mathbb{C}^{6}\right):\right.$

$\left.\sum_{i, j} \omega\left(v_{i}, v_{j}\right)=0\right\}$ 
$S p_{2 n}$,

$S p_{2 n}$

$\mathrm{SO}_{2 n}$,

$F_{4}$,

$F_{4}$,

$F_{4}$,

$$
\mathfrak{g}_{2} \oplus \mathbb{C} \oplus \mathbb{C}\left(Y_{-\alpha_{4}}+Y_{1232}\right) \oplus \bigoplus_{\alpha \in\left\langle 1121,1221,0121, \alpha_{3}\right\rangle}
$$

with $\mathfrak{t} \cap \mathfrak{g}_{2} \oplus \mathbb{C}=\operatorname{Lie}\left(\operatorname{ker}\left(\alpha_{4}+1232\right)\right)$,

$G_{2}$

$G_{2}$

$G_{2}$

$G_{2}$,

$\mathfrak{s p}_{2} \times \mathfrak{s p}_{2 n-2}$,

$\mathfrak{t} \oplus \bigoplus_{\alpha \in \Psi} \mathfrak{g}_{\alpha}$ with $\Psi=\left\langle \pm \alpha_{2}, \ldots, \pm \alpha_{n}, 2 \varepsilon_{1}\right\rangle$,

$\mathfrak{s o}_{2 n-1}$,

$\mathfrak{s p i n}_{9}$,

$\mathfrak{t} \oplus \bigoplus_{\alpha \in \Psi} \mathfrak{g}_{\alpha}$ with $\Psi=\left\langle \pm \alpha_{2}, \alpha_{3}, \pm \alpha_{4}, 1120\right\rangle$,

$\mathfrak{t} \oplus \bigoplus \mathfrak{g}_{\alpha}$ with $\Psi=\Phi^{+} \backslash\left\{\alpha_{2}, \alpha_{1}+\alpha_{2}\right\}$,

$\mathfrak{t} \oplus \bigoplus_{\alpha \in \Psi} \mathfrak{g}_{\alpha}$ with $\Psi=\Phi^{+} \backslash\left\{\alpha_{1}, \alpha_{1}+\alpha_{2}\right\}$,

$\mathfrak{t} \oplus \bigoplus_{\alpha \in \Psi} \mathfrak{g}_{\alpha}$ with $\Psi=\left\{-\alpha_{2}\right\} \cup \Phi^{+} \backslash\left\{\alpha_{1}, \alpha_{1}+\alpha_{2}\right\}$,

$\mathfrak{s l}_{3}$,
$\operatorname{Gr}(2 n ; 2)$

$\left\{(\ell, P) \in \mathbb{P}^{2 n} \times \operatorname{Gr}(2 n ; 2): \ell \subset P\right\}$

$\mathrm{Q}(2 n)$

$E_{6} / P_{6}$

$\mathrm{Cl}\left(G \cdot\left[v_{\lambda-\beta}\right]\right) \subset \mathbb{P}\left(V\left(\omega_{1}\right) \otimes V\left(\omega_{4}\right)\right)$

$\lambda=\omega_{1}+\omega_{4}, \quad \beta=1111$

$\mathrm{Cl}\left(G \cdot=\left[v_{\omega_{4}}+v_{s_{\alpha} s_{\beta}\left(\omega_{4}\right)}\right]\right) \subset \mathbb{P}\left(V\left(\omega_{4}\right)\right)$

$\alpha=\alpha_{4}, \quad \beta=1231$

$G \times{ }_{B} \Sigma_{3}$

$G \times_{B} \Sigma_{2}$

$G_{2} \times P_{P_{1}} \mathbb{P}^{2}$

with $\mathbb{P}^{2}$ compactification of $P_{1} / H=\mathbb{C}^{2}$

$\mathrm{Q}(7)$ action induced by $\mathrm{SO}_{7}$ 's 
Remark. In the first column, we list the group acting on the two-orbit variety designed in the last column. In the second column, we have the corresponding (connected) generic stabilizer. We denote the Grassmannian of $m$-planes in $\mathbb{C}^{n}$ by $\operatorname{Gr}(n ; m)$, the $n$-1-dimensional quadric $\mathrm{Q}(n) \subset \mathbb{P}^{n}$ with the natural action of $S O_{n}$ given by $g \cdot\left[z_{0}: z^{\prime}\right]=\left[z_{0}: g \cdot z^{\prime}\right]$ and by $\Sigma_{k}$ the Hirzebruch surface.

\section{General properties}

We first work out the case of two-orbit $S L_{2}$-varieties.

Proposition 3.1. The two-orbit $S L_{2}$-varieties are $\mathbb{P}^{1} \times \mathbb{P}^{1}$ and $\mathbb{P}^{2}$ with the obvious actions of $S L_{2}$. The respective generic stabilizers are the maximal torus $T$ and its normalizer.

Proof. Recall that an $S L_{2}$-orbit is projective if and only if it is of dimension 0 or 1. Since 3-dimensional $S L_{2}$-orbits are affine this implies that a two-orbit $S L_{2^{-}}$ variety $X$ has dimension 2 . It follows that the maximal torus $T$ fixes at least three points of $X$ ([13, Theorem 25.2]). Thus the generic stabilizer $H$ contains $T$ and so $H$ is either $T$ or its normalizer $H=N_{S L_{2}}(T)$. This, in turn, implies that $G / H$ is affine and so the closed orbit has dimension 1. Now the canonical inclusions $S L_{2} / T \rightarrow \mathbb{P}^{1} \times \mathbb{P}^{1}$ and $S L_{2} / N_{G}(T) \rightarrow \mathbb{P}^{2}$ extend to equivariant birational maps $X \rightarrow P^{1} \times \mathbb{P}^{1}$ or $X \rightarrow \mathbb{P}^{2}$. But since the locus of indeterminacy of such a map has codimension greater or equal than 2 and is $G$-stable it follows that these maps are regular and hence isomorphisms.

From now on, the group $G$ is of rank greater or equal than 2. Let us start by studying locally the two-orbit $G$-variety $X$. Consider $y$ the $B$-fixed point of $X$ and $P$ its stabilizer in $G$. The group $P$ is parabolic and $P^{u} L$ will be its Levi decomposition such that $T \subset L$. By [6, Theorem 1.4], there exists an affine $L$ stable subvariety $Z$ of $X$ such that $\operatorname{dim} Z \geq 1$ and $Z \cap G \cdot y=\{y\}$. Using the fact that $T$-orbits are affine, it is easy to see that an affine $T$-variety containing fixed points also contains 1-dimensional orbits if the action is not trivial. Therefore, there is a generic element $x$ of $X$ such that $\operatorname{codim}_{T} T_{x} \leq 1$. In other words, we have just obtained

Proposition 3.2. The ranks of the algebraic group $G$ and of the generic stabilizer differ at most by 1.

Definition. A two-orbit $G$-variety is said to be of type $I$ if the ranks of $G$ and of the generic stabilizer are equal. Otherwise, it is said of type II.

Suppose that the generic element $x$ is such that the torus $T_{x}^{\circ}$ is a maximal torus of $G_{x}$. Let $L^{\prime}$ be the centralizer in $G$ of $T_{x}^{\circ}$. Then, by the above proposition, $L^{\prime}$ 
is equal to $T$ or is a Levi subgroup of semisimple rank 1 . We are going to prove that the latter possibility can not occur i.e.

Proposition 3.3. The maximal tori of the generic stabilizer are regular tori of the group $G$.

For this, we will need the following

Lemma 3.4. Let $H$ be a subgroup of $G$ and $S$ a torus of $G$. Consider $L$ the identity-component of the centralizer in $G$ of $S$. Then the connected components of $(G / H)^{S}$, the $S$-fixed points of $G / H$, are exactly given by its L-orbits.

Proof. We prove this standard statement on the corresponding tangent spaces. Let $z \in(G / H)^{S}$ such that $G_{z}=H$. We consider the tangent space $T_{z}\left((G / H)^{S}\right)$ at $z$. The lemma follows from the isomorphisms:

$$
T_{z}\left((G / H)^{S}\right) \cong(\mathfrak{g} / \mathfrak{h})^{S} \cong \mathfrak{g}^{S} / \mathfrak{h}^{S} \cong \mathfrak{l} / \mathfrak{l}_{z}
$$

Proof of Proposition 3.3. We proceed by contradiction; suppose that $L^{\prime} \neq T$ and consider the projective $L^{\prime}$-variety $\mathrm{Cl}\left(L^{\prime} \cdot x\right)$ in $X$. Since $T_{x}^{\circ} \neq T$, it is actually a $S L_{2}$-variety with more than two $S L_{2}$-orbits (see Proposition 3.1 ). More precisely, $\mathrm{Cl}\left(L^{\prime} \cdot x\right) \backslash L^{\prime} \cdot x$ is a finite union of closed $L^{\prime}$-orbits of dimension $\leq 1$ all contained in $G \cdot y$ because of Lemma 3.4. Therefore (see also the proof of Proposition 3.1), the $L^{\prime}$-variety $\mathrm{Cl}\left(L^{\prime} \cdot x\right)$ is of dimension 2 .

Let $B_{L^{\prime}}$ be the Borel subgroup of $L^{\prime}$ equal to $B \cap L^{\prime}$ and $U_{L^{\prime}}$ its unipotent radical. Since $\mathrm{Cl}\left(L^{\prime} \cdot x\right) \backslash L^{\prime} \cdot x$ is not connected there exists an element $x^{\prime}$ in $L^{\prime} \cdot x$ fixed by $U_{L^{\prime}}$ (see again the corresponding argument given in the introduction). Thus, $\mathrm{Cl}\left(L^{\prime} \cdot x^{\prime}\right)=L^{\prime} \cdot \mathrm{Cl}\left(B_{L^{\prime}} \cdot x^{\prime}\right)=L^{\prime} \cdot \mathrm{Cl}\left(T \cdot x^{\prime}\right)$. Moreover, $T_{x}^{\circ}$ fixes obviously $x^{\prime}$ so, by Proposition $3.2, T_{x}^{\circ}=T_{x^{\prime}}^{\circ}$. If $y_{1}$ and $y_{2}$ denote the $T$-fixed points in $\mathrm{Cl}\left(T \cdot x^{\prime}\right)$ then $\mathrm{Cl}\left(L^{\prime} \cdot x\right)=L^{\prime} \cdot x \cup L^{\prime} \cdot y_{1} \cup L^{\prime} \cdot y_{2}$. The variety $\mathrm{Cl}\left(L^{\prime} \cdot x\right)$, being 2-dimensional, has at least three $T$-fixed points, (see [13, Theorem 25.2]). Therefore, $y_{1}$ and $y_{2}$ can not be simultaneously fixed by $L^{\prime}$. Suppose that $L^{\prime} \cdot y_{1} \neq y_{1}$ and consider the element $y^{\prime}$ satisfying $\mathrm{Cl}\left(T \cdot y^{\prime}\right)=L^{\prime} \cdot y_{1}$. Then we get

$$
T_{y^{\prime}}^{\circ}=T_{x^{\prime}}^{\circ}, \quad \operatorname{dim}\left(T \cdot x^{\prime}\right)=\operatorname{dim}\left(T \cdot y^{\prime}\right)=1 \quad \text { and } \quad y_{1} \in \mathrm{Cl}\left(T \cdot x^{\prime}\right) \cap \mathrm{Cl}\left(T \cdot y^{\prime}\right) .
$$

It results from the lemma below that $y_{2} \in L \cdot y_{1}$ which is incompatible with the fact that $\mathrm{Cl}\left(L^{\prime} \cdot x\right)$ has more than two $L$-orbits. Thus the assumption $L^{\prime} \neq T$ was absurd - which proves Proposition 3.3.

Lemma 3.5. Let $x$ and $z$ be two elements of a projective G-variety such that

$$
\operatorname{dim} T \cdot x=\operatorname{dim} T \cdot z=1 \quad \text { and } \quad T_{x}^{\circ}=T_{z}^{\circ} .
$$

Let $Z=\mathrm{Cl}(T \cdot x) \cup \mathrm{Cl}(T \cdot z)$. If all $T$-fixed points $Z^{T}$ are contained in a single projective $G$-orbit then $Z^{T}$ consists either of two or of four points. 
Proof. By embedding the given projective $G$-variety $G$-equivariantly into the projective space of a $G$-module, we can write the elements $x$ and $z$ as: $x=\left[v_{\mu_{1}}+\right.$ $\left.\ldots+v_{\mu_{r}}\right]$ with $v_{\mu_{i}} \neq 0$ for all $i$ 's and $z=\left[v_{\nu_{1}}+\ldots+v_{\nu_{s}}\right]$ with $v_{\nu_{j}} \neq 0$ for all $j$ 's. By assumption, the weights $\mu_{i}\left(\right.$ resp. $\left.\nu_{j}\right)$ sit on a same affine line $D_{x}$ (resp. $D_{z}$ ) and moreover,

$$
\operatorname{ker}\left(\mu_{1}-\mu_{r}\right)^{\circ}=T_{x}^{\circ}=T_{z}^{\circ}=\operatorname{ker}\left(\nu_{1}-\nu_{s}\right)^{\circ} .
$$

From these equalities, we can deduce that the lines $D_{x}$ and $D_{z}$ are parallel or equal. The first situation clearly yields four $T$-fixed points in $Z$. Concerning the second one, we have to show that there are actually only two $T$-fixed points in $Z$. For this, we name the weights $\mu_{i}$ and $\nu_{j}$ in order to have $Z^{T}=\left\{\left[v_{\mu_{1}}\right],\left[v_{\mu_{r}}\right],\left[v_{\nu_{1}}\right],\left[v_{\nu_{s}}\right]\right\}$. Since $Z^{T}$ is entirely contained in one single projective $G$-orbit, its elements are all conjugated by the Weyl group $W$ of $G$. And in particular, the weights $\mu_{1}, \mu_{r}, \nu_{1}$ and $\nu_{s}$ are extremal points of the convex hull of $W \cdot \mu_{1}$. Therefore, if $D_{x}=D_{z}$ then the $T$-fixed points of $\mathrm{Cl}(T \cdot x)$ and of $\mathrm{Cl}(T \cdot z)$ must coincide.

As a consequence of Proposition 3.3, we have:

Corollary 3.6. Consider two elements $x$ and $z$ of the dense $G$-orbit of a twoorbit variety such that $T_{x}^{\circ}$ (resp. $T_{z}^{\circ}$ ) is a maximal torus of $G_{x}$ (resp. $G_{z}$ ). Then $z \in N_{G}(T) \cdot x$; in particular, if $x$ and $z$ are $B$-conjugated they are actually $T$ conjugated.

\section{Parabolic induction}

This section is the main step to the classification of two-orbit varieties. It consists essentially in proving that the problem of classifying the two-orbit varieties can be reduced to a subclass of two-orbit varieties called cuspidal, i.e. the two-orbit varieties which can not be obtained by parabolic induction (see section 2).

\subsection{Statements}

Proposition 4.1. If $X$ is obtained by parabolic induction from a pair $(P, Y)$ then as an embedding of its open $G$-orbit it is completely determined (up to isomorphism) by its closed $G$-orbit and by the P-variety $Y$.

Proof. Suppose the parabolic subgroup $P$ contains the Borel subgroup $B$ and consider the Borel subgroup $B^{-}$such that $B \cap B^{-}=T$. By the theory of Luna and Vust on embeddings of homogeneous varieties (see [14]), we know that $X$ is completely determined (up to isomorphism) by its $G$-stable prime divisors and by the $B^{-}$-stable prime divisors of its dense $G$-orbit whose closure in $X$ contains the closed $G$-orbit (see Theorem 8.3 of [loc. cit.]). 
Note that if the closed $G$-orbit of $X$ is a divisor, the proposition follows easily since the birational morphism $G \times_{P} Y \rightarrow X$ is an isomorphism. Therefore, in the rest of the proof, we suppose that it is not the case.

Let $L$ be the Levi subgroup of $P$ such that $T \subset L$ and $B_{L}^{-}$its Borel subgroup such that $B_{L}^{-}=B^{-} \cap L$. Consider the $G$-equivariant morphism $\pi: G \times{ }_{P} Y \rightarrow G / P$. Let $D$ be any $B^{-}$-stable prime divisor of the dense $G$-orbit of $X$ (or equivalently of $\left.G \times{ }_{P} Y\right)$. Then $\pi(D)$ is dense in $G / P$ or it is contained in a $B^{-}$-stable divisor of $G / P$.

Suppose that $\pi(D)$ is dense in $G / P$. Then there exists a $B_{L}^{-}$-stable prime divisor $D_{Y}$ of the dense $P$-orbit of $Y$ such that $B^{-} \cdot D_{Y} \subset D$. Therefore, the closure of $D$ in $X$ contains the closed $G$-orbit of $X$ if and only if the closure of $D_{Y}$ in $Y$ contains the closed $P$-orbit of $Y$. The proposition follows in this case.

Suppose now that $\pi(D)$ is contained in a $B^{-}$-stable divisor of $G / P$. Then the closure of $D$ in $G \times{ }_{P} Y$, say $\widetilde{D}$, is the inverse image by $\pi$ of a $B^{-}$-stable divisor of $G / P$ and the intersection of $\widetilde{D}$ with the closed $G$-orbit of $G \times{ }_{P} Y$ is a divisor $D^{\prime}$. Therefore, the closure of $D$ in $X$ contains the closed $G$-orbit of $X$ if and only if the image of $D^{\prime}$ through $\varphi$ (morphism given by the definition of the pair $(P, Y)$ ) is the closed $G$-orbit of $X$. This ends the proof of the proposition.

We can put an order $\leq$ on the set of induction pairs of $X$, defined naturally, for two pairs $\left(P_{1}, Y_{1}\right)$ and $\left(P_{2}, Y_{2}\right)$, by:

$$
\left(P_{1}, Y_{1}\right) \leq\left(P_{2}, Y_{2}\right) \quad \text { if } P_{1} \subseteq P_{2} \text { and } \varphi_{1}\left(Y_{1}\right) \subseteq \varphi_{2}\left(Y_{2}\right)
$$

where $\varphi_{1}$ and $\varphi_{2}$ are morphisms given by the definition (see section 2) of the induction pairs $\left(P_{1}, Y_{1}\right)$ and $\left(P_{2}, Y_{2}\right)$ respectively.

Theorem 4.2. The set of induction pairs of a two-orbit variety (endowed with the order $\leq)$ has an unique minimal element.

\subsection{Proof of Theorem 4.2}

In order to prove Theorem 4.2, we will give an explicit construction of the minimal induction pair.

Let us consider an induction pair $(P, Y)$ of $X$ and two elements $x$ and $z$ in the dense $G$-orbit of $X$. Suppose that $B \cdot x$ and $B \cdot z$ are closed in $G \cdot x$. This means that $B_{x}$ (resp. $B_{z}$ ) contains a Borel subgroup of $G_{x}$ (resp. $G_{z}$ ) and in particular, $B_{x}$ (resp. $B_{z}$ ) contains a maximal torus of $G_{x}$ (resp. $G_{z}$ ). Let $S_{1} \subset B_{x}$ (resp. $S_{2} \subset B_{z}$ ) be such a torus and $\widetilde{S}_{1} \supset S_{1}$ (resp. $\widetilde{S}_{2} \supset S_{2}$ ) a maximal torus of $B$. Then, by considering the elements $x^{\prime}=b_{1} \cdot x$ and $z^{\prime}=b_{2} \cdot z$ for elements $b_{1}$ and $b_{2}$ of $B$ such that $\widetilde{S}_{1}=b_{1}^{-1} T b_{1}$ and $\widetilde{S}_{2}=b_{2}^{-1} T b_{2}$, one can show that $T_{x^{\prime}}^{\circ}$ (resp. $T_{z^{\prime}}^{\circ}$ ) is a maximal torus of $G_{x^{\prime}}\left(\operatorname{resp} . G_{z^{\prime}}\right)$. Therefore, by Corollary 3.6, $x^{\prime}$ and $z^{\prime}$ are conjugated by an element $n \in N_{G}(T)$. Moreover, because of the choice made 
on $B \cdot x$ and on $B \cdot z$, one can show by a short computation that $n$ is in fact an element of $P$. We have got:

Lemma 4.3. If $X$ is obtained from a pair $(P, Y)$ by parabolic induction then the $B$-orbits which are closed in the dense $G$-orbit are in the same P-orbit of $X$. In particular, they are contained in $\varphi(Y)$ for $\varphi$ a morphism given by the definition of $(P, Y)$.

Let $x$ be a generic element of $X$ such that $B \cdot x$ is closed in $G \cdot x$ and $T_{x}^{\circ}$ is a maximal torus of $G_{x}$. Denote $G_{x}$ by $H$. Let $P_{1}$ be the parabolic subgroup of $G$ generated by $B, H$ and the elements $n$ of $N_{G}(T)$ such that $B n \cdot x$ is closed in $G \cdot x$. By construction, for all pairs $(P, Y)$, we have: $P_{1} \subset P$ and $X_{1}=\operatorname{Cl}\left(P_{1} \cdot x\right) \subset$ $\varphi(Y)$. However, $X_{1}$ may not be a two-orbit $P_{1}$-variety. More precisely, we have: $X_{1} \cap G \cdot x=P_{1} \cdot x$ but an analogous equality may not hold for $X_{1} \cap G \cdot y(y$ a $T$-fixed element of the closed orbit in $\left.X_{1}\right)$. So, instead of $P_{1}$, we have to consider the parabolic subgroup $P_{H}$ of $G$ generated by $P_{1}$ and the elements $w \in W$ such that $w \cdot y \in X_{1}$. Thus, $X_{H}=\mathrm{Cl}\left(P_{H} \cdot x\right)=\mathrm{Cl}\left(P_{H} \cdot X_{1}\right)$ has two $P_{H}$-orbits with $P_{H} \subset P$ and $X_{H} \subset \varphi(Y)$.

Finally, let us consider the normalization $\pi: \widetilde{X}_{H} \rightarrow X_{H}$ of $X_{H}$. The pair $\left(P_{H}, \widetilde{X}_{H}\right)$ is the required element.

Proposition 4.4. The pair $\left(P_{H}, \widetilde{X}_{H}\right)$ is an induction pair; this is the minimal element for the set of induction pairs of $X$.

We already know (see section 2 ) that the variety $\widetilde{X}_{H}$ is a two-orbit $P_{H \text {-variety }}$ and that $\pi$ is bijective (so in particular, $\left.\pi\left(\widetilde{X}_{H}\right)=X_{H}\right)$. Therefore by construction, the minimal condition is fulfilled by $\left(\widetilde{X}_{H}, P_{H}, \pi\right)$, that is $\pi\left(\widetilde{X}_{H}\right) \subset \varphi(Y)$ and $P_{H} \subset P$ for any induction pair $(P, Y, \varphi)$ of $X$. So we are left to prove that the radical of $P_{H}$ acts trivially on $\widetilde{X}_{H}$. For this, we use the following

Proposition 4.5. Let $Y$ be a projective $Q$-variety with two $Q$-orbits, $Q$ being a parabolic subgroup of $G$ containing $B$. Consider an element $z$ of its dense $Q$-orbit such that $B \cdot z$ is closed in $Q \cdot z$ and $T_{z}^{\circ}$ is a maximal torus of $Q_{z}$. Suppose that the radical of $Q$ does not act trivially on $Y$. Then the orbit $L \cdot z$ is complete for a Levi subgroup $L$ of $Q$ containing the torus $T$.

Proof of Proposition 4.4. According to Proposition 4.5 applied to $\widetilde{X}_{H}, P_{H}=P_{H}^{u} L$ and $x \in X_{H}$, if we prove that $L \cdot x$ is not complete, Proposition 4.4 will follow. If $T_{x}^{\circ} \neq T$, that $L \cdot x$ is not complete is obvious. Consider then the other case: $T_{x}^{\circ}=T$. If $\alpha$ is a simple root of $P_{H}$ such that $U_{\alpha} \not \subset G_{x}$ then (because of the assumption made on $B \cdot x) s_{\alpha} B s_{\alpha} \cap G_{x}$ contains a Borel subgroup of $G_{x}$. In other words, $B s_{\alpha} \cdot x$ is closed in $P_{H} \cdot x$ and by definition of $P_{H}$, it means that $S L_{2}(\alpha) \subset L$. To conclude that $L \cdot x$ is not complete, we only need to observe that 
$\mathrm{Cl}(L \cdot x)$ contains a $T$-fixed point of the closed $G$-orbit of $X$ (because $\mathrm{Cl}\left(U_{\alpha} \cdot x\right)$ does by Corollary 3.6).

Proof of Proposition 4.5. First of all, note that the radical of $Q$ acts trivially on the closed $Q$-orbit of $Y$. So the proposition relies only on the dense $Q$-orbit.

Let us start with the case: $T_{z}^{\circ} \neq T$. Thus $L \cdot z$ is not complete. Let $\beta \in \Phi^{+}$ be such that $U_{\beta} \not \subset Q_{z}$. Such a root exists otherwise $Y$ will have two $B$-fixed points given by the $T$-fixed points of $\mathrm{Cl}(T \cdot z)$. Consider the 2-dimensional variety $X_{\beta}=\mathrm{Cl}\left(U_{\beta} T \cdot z\right)$ and denote by $T_{X_{\beta}}(\subset \operatorname{ker} \beta)$ its generic stabilizer in $T$. To get our result, we are going to prove that $T_{X_{\beta}}^{\circ}$ contains the identity-component $Z(L)^{\circ}$ of the center of $L$. Indeed, it will imply that $Z(L)^{\circ} \subset \operatorname{ker} \beta$ hence $U_{\beta} \not \subset Q^{u}$.

Let $y_{1}$ and $y_{2}$ be the $T$-fixed points of $\mathrm{Cl}(T \cdot z)$. If $y_{i}(i=1,2)$ is not fixed by $U_{\beta}$, we denote by $y_{i}^{\prime}$ the other $T$-fixed point in $\mathrm{Cl}\left(U_{\beta} \cdot y_{i}\right)$; otherwise, we set $y_{i}^{\prime}=y_{i}$. The points $y_{1}^{\prime}$ and $y_{2}^{\prime}$ are distinct; this follows easily from Lemma 3.5 and from the fact that $T_{z}^{\circ}$ is regular.

The variety $X_{\beta}^{U_{\beta}}$, being connected and containing the two distinct elements $y_{1}$ and $y_{2}$, is 1-dimensional.

Claim. There exist $z_{1}, \ldots, z_{r}$ and $\rho_{1}, \ldots, \rho_{r+1}(r \geq 1)$ in $X_{\beta}^{U_{\beta}}$ such that $\operatorname{dim} T$. $z_{i}=1$ and such that $\rho_{i}, \rho_{i+1}$ are the $T$-fixed points in $\operatorname{Cl}\left(T \cdot z_{i}\right)$. Moreover, if $y_{i}=y_{i}^{\prime}(i=1,2)$ then $r \geq 2$.

To get the elements $z_{i}$ and $\rho_{i}$, it suffices to consider the convex hull of the support of $X_{\beta}$; the elements $z_{i}$ (resp. $\rho_{i}$ ) have as support the edges (resp. vertices) of this polytope.

Because of the claim, we have now at hand at least two elements $u$ and $v$ simultaneously in the closed $Q$-orbit of $Y$ and in $X_{\beta}$ such that: $\operatorname{dim}(T \cdot u)=1=$ $\operatorname{dim}(T \cdot v)$ and the cardinality of $\mathrm{Cl}(T \cdot u) \cap \mathrm{Cl}(T \cdot v)$ is 1 . Therefore $\left(T_{u} \cap T_{v}\right)^{\circ}$ is of codimension 2 hence equal to $T_{X_{\beta}}^{\circ}$. But since $Z(L)$ acts trivially on the closed $Q$-orbit, we end up with the required inclusion: $Z_{L}^{\circ} \subset T_{X_{\beta}}^{\circ}$.

Assume now that $T_{z}^{\circ}=T$ and that $L \cdot z$ is not closed. Therefore, there exists at least one positive root, say $\alpha$, in the root system of $(L, L)$ such that $U_{\alpha} \not \subset Q_{z}$. Recall that we want to prove that the radical of $Q$ acts trivially on $Q \cdot z$. But since the center of $L$ is already contained in $Q_{z}$, we only need to prove that the unipotent radical of $Q$ acts trivially on $Q \cdot z$. To do so, we proceed by contradiction: suppose there exists $\beta \in \Phi^{+}, \beta \neq \alpha$ such that $U_{\beta} \subset Q^{u}$ and $U_{\beta} \not \subset Q_{z}$. Then the variety $X_{\beta, \alpha}=\mathrm{Cl}\left(U_{\beta} U_{\alpha} \cdot z\right)$ is 2-dimensional and contains a dense $T$-orbit; denote by $T_{X_{\beta}}$ its generic stabilizer in $T$.

If $y_{1}$ and $y_{2}$ are the $T$-fixed points (distinct from $z$ ) of $\mathrm{Cl}\left(U_{\alpha} \cdot z\right)$ and of $\mathrm{Cl}\left(U_{\beta} \cdot z\right)$ respectively, then $y_{1}$ and $y_{2}$ are necessarily distinct (as they do not have the same support). If $U_{\beta} \cdot y_{1} \neq y_{1}$, we denote by $y_{1}^{\prime}$ the other $T$-fixed point of $\operatorname{Cl}\left(U_{\beta} \cdot y_{1}\right)$; otherwise, we set $y_{1}^{\prime}=y_{1}$. Then the variety $X_{\beta, \alpha}^{U_{\beta}}$ is 1-dimensional, since it contains the distinct points $y_{1}^{\prime}$ and $y_{2}$. But $y_{1}$ and $y_{2}$ must be the only $T$-fixed points simultaneously in the closed $Q$-orbit of $Y$ and in $X_{\beta, \alpha}$ otherwise with the same 
arguments used in the first case $\left(T_{z}^{\circ} \neq T\right)$, we will have: $Z(L)^{\circ} \subset T_{X_{\beta}}$. But this inclusion is not true as $Z(L)$ does not act trivially on $U_{\beta} \cdot z$. So we can conclude that $U_{\beta}$ must fix $y_{1}$ and that there exists an element $u$ in the closed $Q$-orbit of $Y$ such that: $X_{\beta, \alpha}^{U_{\beta}}=\mathrm{Cl}(T \cdot u)$.

Let $y_{3} \neq y_{1}$ be the other $T$-fixed point of the 1 -dimensional variety $\operatorname{Cl}\left(U_{-\alpha} \cdot y_{1}\right)$.

To summarize, we have constructed two 1-dimensional subvarieties $X_{\beta, \alpha}$ and $\mathrm{Cl}\left(U_{-\alpha} \cdot y_{1}\right)$ of the closed $Q$-orbit such that $y_{1}$ is their only common $T$-fixed point. According to Lemma 3.5, this yields the contradiction: $\left(T_{u} \cap \operatorname{ker} \alpha\right)^{\circ}=T_{X_{\beta}}^{\circ}$.

\section{Two-orbit varieties of type I}

In this section, the two-orbit $G$-variety $X=\mathrm{Cl}(G \cdot x)=G \cdot x \cup G \cdot y$ is of type I, i.e. $T_{x}^{\circ}=T$. Recall (see section 2 ) that a two-orbit variety is projective. So we can embed $X$ in $\mathbb{P}(V)$ with $V$ a finite dimensional $G$-module. We identify $X$ with its image in $\mathbb{P}(V)$. The elements $x$ and $y$ of $X$ can be written as $x=\left[v_{\mu}\right]$ and $y=\left[v_{\lambda}\right]$ with weight vectors $v_{\mu}$ and $v_{\lambda}$. Since $X$ contains only one projective $G$-orbit the module $V$ can be chosen to be of the form $V(\lambda) \oplus \oplus_{\nu} V(\nu)$ with $\nu$ in the convex hull of $W \cdot \lambda$.

Given $\beta \in \Phi^{+}$such that $U_{\beta} \not \subset G_{x}$ (such a root exists otherwise $B \subset G_{x}$ ), consider $z=\left[v_{\mu}+v_{\mu+\beta} \cdots+v_{\mu+k \beta}\right] \in U_{\beta} \cdot x$. With a judicious choice of $x$, (for instance $\mu$ dominant), one shows easily that $\left[v_{\mu+k \beta}\right] \in G \cdot y$. So finally, we can choose $x$ such that $\lambda=\mu+k \beta$ (take a $W$-conjugate of the previous $x$ if necessary).

The support of a root $\beta$, denoted by $\operatorname{supp} \beta$, is defined to be the set of simple roots which occur in presentation of $\beta$ as a linear combination of simple roots.

Proposition 5.1. If $X=G \cdot x \cup G \cdot y$ is cuspidal then $G$ is simple. Moreover $\operatorname{supp} \beta=\Delta$ with $\beta \in \Phi^{+}$such that $U_{\beta} \not \subset G_{x}$ and $y=\left[v_{\lambda}\right] \in \operatorname{Cl}\left(U_{\beta} \cdot x\right)$.

Proof. Recall from the construction of the minimal induction pair (see section 4.2) that since $X$ is cuspidal, $G$ must be generated by the parabolic subgroup $P_{1}$ and the elements $w \in W$ such that $w \cdot\left[v_{\lambda}\right] \in X_{1}$. Recall that $P_{1}$ is spanned by $H, B$ and the elements $n \in N_{G}(T)$ such that $B n \cdot x$ is closed in $G \cdot x$ and $X_{1}=\operatorname{Cl}\left(P_{1} \cdot x\right)$.

If $G=G_{1} \times \cdots \times G_{r}$ with $G_{i}$ simple and $\Delta=\Delta_{1} \times \cdots \times \Delta_{r}$ with $\Delta_{i}$ associated to $G_{i}$, we are going to show that $H=H_{1} \times \cdots \times H_{r}$ with $H_{i}=G_{i}$ for all $i \neq i_{0}$ and $i_{0}$ such that $\operatorname{supp} \beta \subset \Delta_{i_{0}}$. First of all, note that if $\alpha \notin \operatorname{supp} \beta$, then $U_{\alpha} \subset G_{x}$. Indeed, $\mu+\ell \alpha(\ell>0)$ is not of shape $\lambda-\sum_{\gamma \in \Delta} n_{\gamma} \cdot \gamma$, (weights of $V$ ) since $\lambda=\mu+k \beta$ (see the choice of $x$ made above). Moreover, if $\alpha \in \Delta \backslash \Delta_{i_{0}}$ then with the above description of $G$ (given by the cuspidal condition), we must have $U_{-\alpha} \subset G_{x}$. Thus: $U_{ \pm \alpha} \subset G_{x}$, for all $\alpha \in \Delta \backslash \Delta_{i_{0}}$. The acting group $G$ can then be assumed to be simple.

To obtain the second assertion, consider the parabolic subgroup $P \supset B$ associated to $\operatorname{supp} \beta$ and the $P$-variety $Z=X \cap \mathbb{P}\left(\bigoplus_{\nu} V_{\nu}\right)$ for $\nu=\lambda-\sum n_{\alpha} \alpha$ 
with $\alpha \in \operatorname{supp} \beta$ and $n_{\alpha} \geq 0$. Then by construction, if $\operatorname{supp} \beta \neq \Delta,(P, Z)$ is an induction pair of $X$.

From now on, $G$ will be simple and $x$ will satisfy the above conditions as well as the two following ones. Consider $x^{\prime}=\left[v_{\mu^{\prime}}\right]$ another generic element of $X$ and $\beta^{\prime} \in \Phi^{+}$such that $U_{\beta^{\prime}} \not \subset G_{x^{\prime}}$. Suppose that $\left[v_{\mu^{\prime}+k^{\prime} \beta^{\prime}}\right] \in \mathrm{Cl}\left(U_{\beta^{\prime}} \cdot x^{\prime}\right) \cap G \cdot y$ for $k^{\prime}$ a positive integer. The first condition we require on $x$ is that $k$ must be smaller or equal than $k^{\prime}$. The second one is that $\mu$ must be greater than $\mu^{\prime}$, for $\mu$ and $\mu^{\prime}$ comparable, if $k=k^{\prime}$ and $\mu^{\prime}+k^{\prime} \beta^{\prime}=\lambda$.

Consider a second positive root $\gamma \neq \beta$ such that $U_{\gamma} \not \subset G_{x}$ (there exists at least one such a root which is simple). Denote by $L$ the Levi subgroup associated to $\beta$ and $\gamma$, i.e. $L$ is the centralizer in $G$ of $(\operatorname{ker} \beta \cap \operatorname{ker} \gamma)^{\circ}$. Then we have

Lemma 5.2. The normalization of the L-variety $\mathrm{Cl}(L \cdot x)$ is a cuspidal two-orbit $L$-variety.

Proof. From Lemma 3.4, we have $L \cdot x=\mathrm{Cl}(L \cdot x) \cap G \cdot x$ and $\mathrm{Cl}(L \cdot x) \backslash L \cdot x$ consists of a finite union of complete $L$-orbits. Thus, if $\mathrm{Cl}(L \cdot x)$ does not have two $L$-orbits, we will have $(L \cdot x)^{U_{L}} \neq \emptyset$ for $U_{L}$ an unipotent maximal subgroup of $L$ (again the same argument as in the introduction). It will follow that $\mathrm{Cl}(L \cdot x)=L \cdot x$. But this equality can not hold since $\left[v_{\lambda}\right] \in \mathrm{Cl}(L \cdot x) \backslash L \cdot x$. Therefore, $\mathrm{Cl}(L \cdot x)$ has exactly two $L$-orbits.

As we can not find any proper parabolic subgroup $P$ such that $P^{u} \subset L_{x} \subset P$ (because $\left.U_{ \pm \beta}, U_{ \pm \gamma} \not \subset G_{x}\right)$, the normalization of $\mathrm{Cl}(L \cdot x)$ has to be cuspidal.

By this procedure, we have constructed two-orbit varieties for some subgroups $L$ of $G$ of semisimple rank 2. Thus, once we know what the two-orbit varieties are, for all simple groups of rank 2, we will know in particular $L_{x}$ (for all $L$ 's as above) hence $G_{x}$.

Let us start with determining the cuspidal two-orbit varieties in the rank 2 case. For this, we need two technical lemmas. Let $\Lambda$ be the convex hull of the support of the variety $\mathrm{Cl}\left(U_{\gamma} U_{\beta} \cdot x\right)$. We have the following picture and notation in the weight lattice $\mathcal{X}$ of $G$.

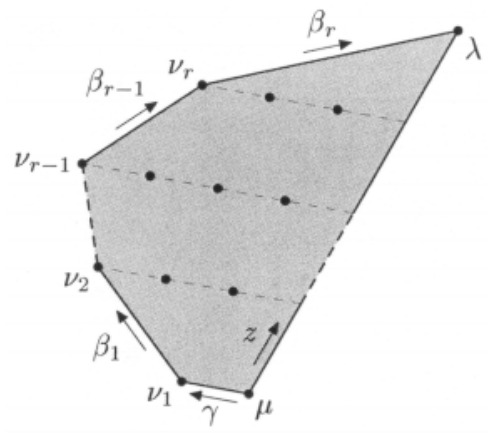


Lemma 5.3. The extremal points $\nu_{i}$ of $\Lambda$ are $W$-conjugated to $\lambda$ and the $\beta_{i}$ 's are some roots of $\Phi$.

Proof. First of all, note that the points $\left[v_{\nu_{i}}\right]$ and the elements $\left[v_{i}\right]$ whose support is $\left[\nu_{i}, \nu_{i+1}\right] \cap \mathcal{X}$ are contained in $\operatorname{Cl}\left(U_{\gamma} U_{\beta} \cdot x\right)$.

Consider the torus $T_{\left[v_{i}\right]}^{\circ}=\operatorname{ker}\left(\nu_{i}-\nu_{i+1}\right)^{\circ}$. Given a maximal torus $T^{\prime}$ of $G_{\left[v_{i}\right]}$ (hence of $G$ in the case of a two-orbit variety of type I) containing $T_{\left[v_{i}\right]}^{\circ}$ and assume that $T_{\left[v_{i}\right]}^{\circ}$ is regular. Then we will have: $C_{G}\left(T_{\left[v_{i}\right]}^{\circ}\right)=T^{\prime}$ hence $T=T^{\prime}$ and thus $T$ will fix $\left[v_{i}\right]$. But this is impossible since the weights $\nu_{i}$ are all distinct. Therefore, the torus $T_{\left[v_{i}\right]}^{\circ}$ is singular and the $\beta_{i}$ 's are some roots.

Let us prove now the first assertion of the lemma. If $\Lambda$ has more than three extremal points then the cardinality of the support of $\left[v_{i}\right]$ is less than the cardinality of $\operatorname{supp} x$. So by the minimality assumption made on $x$, we must have $\left[v_{i}\right] \in G \cdot y$ and thus $\left[v_{\nu_{i}}\right] \in G \cdot y$. If $\Lambda$ has three extremal points, the assumed maximality of the weight $\mu\left(x=\left[v_{\mu}\right]\right)$ forces $\nu_{1}$ to be in $W \cdot \lambda$.

Lemma 5.4. Let $\delta$ be a positive root such that $\left[v_{s_{\gamma^{\prime}}(\lambda)}\right] \in \operatorname{Cl}\left(U_{\delta} \cdot x\right)$, for $\gamma^{\prime} \in \Delta$. Suppose that: if there exists $r \geq 0$ such that $\mu+r \gamma^{\prime}$ is extremal as a weight of $V$, we must have $\mu+r \gamma^{\prime}=s_{\alpha}(\lambda)$ for $\alpha \in \Delta$. Then, $U_{\gamma^{\prime}} \subset G_{x}$ if $\left(\mu, \gamma^{\prime}\right) \geq 0$ and $U_{-\gamma^{\prime}} \subset G_{x}$ if $\left(\mu, \gamma^{\prime}\right) \leq 0$.

Proof. If there is no $r>0$ such that $\mu+r \gamma^{\prime}$ is extremal as weight of $V$ then by Lemma 5.3, we must have $U_{\gamma^{\prime}} \subset G_{x}$ if $\left(\mu, \gamma^{\prime}\right) \geq 0$. If $\left(\mu, \gamma^{\prime}\right) \leq 0$ and $U_{-\gamma^{\prime}} \not \subset G_{x}$ then $\mathrm{Cl}\left(U_{-\gamma^{\prime}} \cdot x\right)$ contains a $T$-fixed point of $G \cdot y$ and so does $\mathrm{Cl}\left(U_{\gamma^{\prime}} \cdot x\right)$ - which is absurd.

Suppose now that $U_{\gamma^{\prime}} \not \subset G_{x}$ and that $\left(\mu, \gamma^{\prime}\right) \geq 0$. Let $x^{\prime}=\varepsilon_{\delta}(1) \cdot x \in U_{\delta} \cdot x$, $\varepsilon_{\delta}$ being the natural map associated to $\delta$ from $\mathbb{C}$ to $U_{\delta}$. Then by assumption $x^{\prime}=\left[v_{\mu}+\cdots+v_{s_{\gamma^{\prime}}(\lambda)}\right]$. The study of $\operatorname{supp}\left(\varepsilon_{\beta}(1) \cdot x^{\prime}\right)$ gives raise to a $j>0$ such that

$$
-\frac{Y_{\beta}^{n_{j}}}{n_{j} !} \cdot v_{\mu+j \delta}=s Y_{\gamma^{\prime}} \cdot v_{s_{\gamma^{\prime}}(\lambda)} \quad \text { for } s \in \mathbb{C} \backslash\{0\} \text { and } n_{j} \geq 0
$$

Recall (see section 2) that $Y_{\beta}$ denotes an element of $\mathfrak{g}_{\beta} \backslash\{0\}$. Furthermore, consider the support of the variety $\operatorname{Cl}\left(T \cdot \exp \left(Y_{\beta}+s Y_{\gamma^{\prime}}\right) \cdot x^{\prime}\right)$. The points $s_{\alpha}(\lambda), s_{\gamma^{\prime}}(\lambda)$ are extremal points of this support but $\lambda$ is not; otherwise, we will have a gap in this support since the weight $\gamma^{\prime}+s_{\gamma^{\prime}}(\lambda)$ is missing by equality (1). It implies that $\left[s_{\alpha}(\lambda), s_{\gamma^{\prime}}(\lambda)\right]$ must be an edge of this support - which contradicts Lemma 5.3 since $s_{\alpha}(\lambda)-s_{\gamma^{\prime}}(\lambda)$ is not a root, $\alpha$ and $\gamma^{\prime}$ being simple. The first assertion of the lemma follows.

If $\left(\mu, \gamma^{\prime}\right) \leq 0$, we can go back to the positive case with the element $s_{\gamma^{\prime}} \cdot x$.

Let us show how we can apply these two lemmas to get the two-orbit varieties in the rank 2 case, through the following 
Example. Suppose $G$ is of type $B_{2}$. Then there are two possibilities for $\beta$ : $\beta=$ $\alpha_{1}+\alpha_{2}$ or $\beta=\alpha_{1}+2 \alpha_{2}$. Let us compute $\mathfrak{g}_{x}$ in case $\beta=\alpha_{1}+\alpha_{2}$.

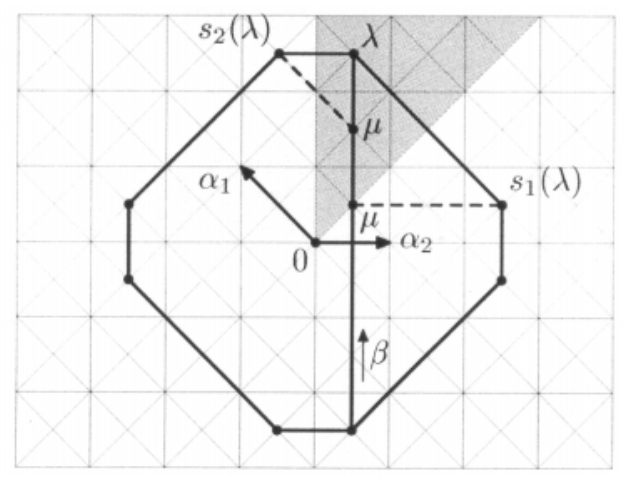

If $U_{\alpha_{1}} \not \subset G_{x}$ then $\alpha_{2}$ satisfies the conditions of Lemma 5.4 with $\left(\mu, \alpha_{2}^{\vee}\right) \geq 0$. Therefore $U_{\alpha_{2}} \subset G_{x}$. Let us show that $U_{\alpha_{1}+2 \alpha_{2}} \subset G_{x}$. If there does not exist $r>0$ such that $\mu+r\left(\alpha_{1}+2 \alpha_{2}\right)$ is extremal then this inclusion is given by Lemma 5.3. Otherwise, we will have: $\mu+r \alpha=s_{1}(\lambda)$. A simple computation leads to: $2\left(\lambda, \alpha_{1}^{\vee}\right)=\left(\lambda, \alpha_{2}^{\vee}\right)$. But in this latter case, $\mu$ does not satisfy the good conditions; in particular, " $k$ " is not minimal. We have obtained the pair: $\left(S O_{5}, \mathfrak{g}_{x}=\mathfrak{t} \oplus \mathfrak{g}_{\alpha_{2}} \oplus \mathfrak{g}_{\alpha_{1}+2 \alpha_{2}}\right)$.

If $U_{\alpha_{1}} \subset G_{x}$ then $U_{\alpha_{2}} \not \subset G_{x}$ and we have $\left(\mu, \pm \alpha_{1}\right)=0$. Thus by Lemma 5.4, we get $U_{ \pm \alpha_{1}} \subset G_{x}$. It follows from Lemma 5.3 that $U_{\alpha_{1}+2 \alpha_{2}} \subset G_{x}$. Therefore, we have obtained the pairs: $\left(S O_{5}, \mathfrak{s o}_{4}\right)$ and $\left(S O_{5}, \mathfrak{g}_{x}=\mathfrak{t} \oplus \mathfrak{g}_{ \pm \alpha_{1}} \oplus \mathfrak{g}_{\alpha_{1}+2 \alpha_{2}}\right)$.

By the procedure given in this example, we get the two-orbit varieties for the rank 2 case. More precisely, we obtain a description of the root system $\Phi\left(G_{x}\right)$ of $G_{x}$.

Lemma 5.5. Let $\gamma \in \Phi^{+}$, we have the following:

(i) if $\langle\gamma, \beta\rangle$ is of type $A_{1} \times A_{1}$ then $\pm \gamma \in \Phi\left(G_{x}\right)$;

(ii) if $\langle\gamma, \beta\rangle$ is of type $A_{2}$ then $\pm \gamma \in \Phi\left(G_{x}\right)$ or $\pm s_{\gamma}(\beta) \in \Phi\left(G_{x}\right)$;

(iii) if $\langle\gamma, \beta\rangle$ is of type $B_{2}$ with $\beta=\varepsilon_{1}+\varepsilon_{j}$ then $\Phi\left(G_{x}\right) \cap\langle\gamma, \beta\rangle=\left\{\varepsilon_{1}-\varepsilon_{j}, \varepsilon_{1}\right\}$;

(iv) if $\langle\gamma, \beta\rangle$ is of type $B_{2}$ with $\beta=\varepsilon_{1}$ then $\Phi\left(G_{x}\right) \cap\langle\gamma, \beta\rangle \supset\left\{\varepsilon_{1}+\varepsilon_{j}, \pm\left(\varepsilon_{1}-\varepsilon_{j}\right)\right\}$ or $\Phi\left(G_{x}\right) \cap\langle\gamma, \beta\rangle=\left\{\varepsilon_{1}+\varepsilon_{j}, \varepsilon_{j}\right\}$.

By applying this statement to a positive root $\beta$ of maximal support and to any other positive root, we get the desired $(G, \mathfrak{h})$. Let us work out two examples to understand how it works.

Example. Suppose $G$ is of type $A_{n}$. Then $\beta=\alpha_{1}+\cdots+\alpha_{n}$. Since $\left\langle\alpha_{i}, \beta\right\rangle$ is of type $A_{1} \times A_{1}$, by Lemma 5.5-(i), we have $\pm \alpha_{i} \in \Phi\left(G_{x}\right)$ for $2 \leq i \leq n-1$. 
Moreover, Lemma 5.5-(ii) yields: $\pm \alpha_{1} \in \Phi\left(G_{x}\right)$ or $\pm \alpha_{n} \in \Phi\left(G_{x}\right)$. This gives the pair $\left(S L_{n+1}, \mathfrak{g l}_{n}\right)$.

Suppose $G$ is of type $B_{n}$ and $\beta=\varepsilon_{1}$. Applying Lemma 5.5-(i) and Lemma 5.5(ii) respectively, we get $\left\{ \pm \alpha_{2}, \ldots, \pm \alpha_{n}\right\} \subset \Phi\left(G_{x}\right)$ and respectively $\left\{\varepsilon_{1}+\varepsilon_{i}\right.$ : $i \geq 2\} \subset \Phi\left(G_{x}\right)$ with $\pm\left(\varepsilon_{1}-\varepsilon_{i}\right) \in \Phi\left(G_{x}\right)$ or $\varepsilon_{i} \in \Phi\left(G_{x}\right)$ for all $i \geq 2$. If $\pm \alpha_{1} \notin \Phi\left(G_{x}\right)$ then $\left\{ \pm \alpha_{1}, \ldots, \pm \alpha_{n-1}, \alpha_{n-1}+2 \alpha_{n}\right\} \subset \Phi\left(G_{x}\right)$. Therefore, we obtain the two pairs $\left(S O_{2 n+1}, \mathfrak{s o}_{2 n}\right)$ and $\left(S O_{2 n+1}, \mathfrak{g}_{x}=\bigoplus_{\alpha \in \Psi} \mathfrak{g}_{\alpha} \oplus \mathfrak{t}\right)$ where $\Psi=$ $\left\langle \pm \alpha_{1}, \ldots, \pm \alpha_{n-1}, \alpha_{n-1}+2 \alpha_{n}\right\rangle$. If $\pm \alpha_{1} \notin \Phi\left(G_{x}\right)$, we get the pair $\left(S O_{2 n+1}, \mathfrak{g}_{x}=\right.$ $\left.\bigoplus_{\alpha \in \Psi} \mathfrak{g}_{\alpha} \oplus \mathfrak{t}\right)$, with $\Psi=\left\langle \pm \alpha_{2}, \ldots, \pm \alpha_{n-1}, \alpha_{n}, \varepsilon_{1}+\varepsilon_{n}\right\rangle$.

\section{Two-orbit varieties of type II}

In this section, the two-orbit $G$-variety $X=\mathrm{Cl}(G \cdot x)$ is of type II, that is, by definition, $T_{x}^{\circ} \neq T$. We embed $X$ in $\mathbb{P}(V)$ with $V$ a finite $G$-module as in the previous section. Then the generic element $x$ can be written as $\left[v_{\lambda_{0}}+\cdots+v_{\lambda_{r+1}}\right]$ with the $\lambda_{i}$ 's sitting on a same affine line, say $D_{x}$ (because of Proposition 3.2). We order the weights $\lambda_{i}$ of $\operatorname{supp} x$ in such a way that $\operatorname{Cl}(T \cdot x)=T \cdot x \cup\left\{\left[v_{\lambda_{0}}\right],\left[v_{\lambda_{r+1}}\right]\right\}$. As elements of $G \cdot y,\left[v_{\lambda_{0}}\right]$ and $\left[v_{\lambda_{r+1}}\right]$ are $W$-conjugate, i.e. there exists $w \in W$ such that $\lambda_{0}=w\left(\lambda_{r+1}\right)$. We choose $x$ such that $\lambda_{0}=\lambda$ and satisfying the following condition of minimality: if $x^{\prime}=\left[v_{\lambda}+\cdots+v_{w^{\prime}(\lambda)}\right]$ is another generic element then $w<w^{\prime}$ if $w$ and $w^{\prime}$ are comparable.

Let $\alpha$ be a simple root such that $s_{\alpha} w<w$ and $U_{\alpha} \not \subset G_{x}$. Take for instance $\alpha$ such that $(w(\lambda), \alpha)<0$. Consider the variety $X_{\alpha}=\mathrm{Cl}\left(T U_{\alpha} \cdot x\right)$ and in particular, the convex hull $\Lambda$ of its support pictured below in the weight lattice $\mathcal{X}$ of $G$.

Similarly as for Lemma 5.3, we get:

Lemma 6.1. The elements $y_{i} \in X_{\alpha}$ whose support sits on the line $D_{i}$ belong to the closed $G$-orbit of $X$. The directions of the affine lines $D_{i}$ are given by roots $\beta_{i}$. In particular, the extremal points $\nu_{i}$ are $W$-conjugated and $\operatorname{supp} y_{i}=\left[\nu_{i}, \nu_{i+1}\right] \cap \mathcal{X}$.

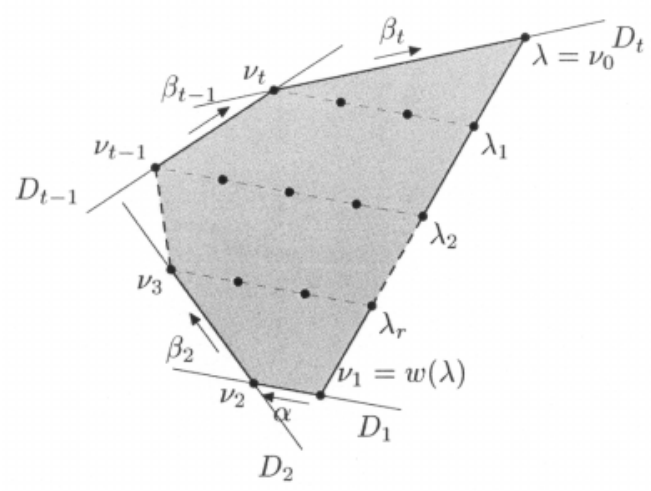


The roots $\beta_{i}$ span a root system of $\operatorname{rank} 2$; let $\{\alpha, \beta\}$ be a basis of this root system.

Corollary 6.2. $w=s_{\alpha} s_{\beta}$.

Proof. We know that $s_{\alpha} w<w$ and that $\lambda-w(\lambda)$ can not be, up to a scalar, a root because $T_{x}^{\circ}=\operatorname{ker}(\lambda-w(\lambda))^{\circ}$ is regular (see Proposition 3.3). So we can assume $\langle\alpha, \beta\rangle$ to be of type $G_{2}$ with $w=\left(s_{\alpha} s_{\beta}\right)^{2}$ - the case $w=w_{0}$ being easily ruled out.

Consider the convex hull $\Lambda$ (see the corresponding picture). Since supp $y_{i}=$ $\left[\nu_{i}, \nu_{i+1}\right] \cap \mathcal{X}$, there exists a weight $\nu \in D_{x}$ such that $\lambda-\beta_{t}=\nu+k \alpha, k \geq 0$. In other words, if $D_{\alpha, \lambda-\beta_{t}}$ denotes the line of direction $\alpha$ passing through $\lambda-\beta_{t}$, we must have:

$$
D_{x} \cap D_{\alpha, \lambda-\beta_{t}} \cap \mathcal{X} \neq \emptyset .
$$

If $w=\left(s_{\alpha} s_{\beta}\right)^{2}$ with $\langle\alpha, \beta\rangle$ of type $G_{2}$ then $\beta_{t}=\beta$ or $\beta_{t}=\beta+\alpha$. But (2) forces $(\lambda, \alpha) /((\lambda, \alpha)+(\lambda, \beta))$ to be an integer - which can not occur because $(\lambda, \alpha) \cdot(\lambda, \beta) \neq 0$ since $T_{x}^{\circ}$ is regular.

Using this corollary and the same arguments given in the proof of Proposition 5.1, we get:

Proposition 6.3. If $X$ is cuspidal of type II then $G$ is simple or of type $A_{1} \times A_{1}$. Furthermore, $\operatorname{supp} \beta \cup\{\alpha\}=\Delta$.

From now on $G$ will be assumed to be simple or of type $A_{1} \times A_{1}$. Let $L$ be the Levi subgroup associated to $\alpha$ and $\beta$ and $\mathfrak{l}$ its Lie algebra.

Proposition 6.4. (i) If $\langle\alpha, \beta\rangle$ is of type $A_{1} \times A_{1}$ then $\mathfrak{l}_{x}=\mathfrak{t}^{\prime} \oplus \mathbb{C}\left(Y_{-\alpha}+Y_{\beta}\right) \oplus$ $\mathbb{C}\left(Y_{\alpha}+Y_{-\beta}\right)$.

(ii) Otherwise, $\mathfrak{l}_{x}=\mathfrak{t}^{\prime} \oplus \mathbb{C}\left(Y_{-\alpha}+Y_{s_{\alpha}(\beta)}\right) \oplus \bigoplus_{\gamma \in \Phi^{+} \cap\langle\alpha, \beta\rangle} \mathfrak{g}_{\gamma}$.

Here, $\mathfrak{t}^{\prime}$ is the kernel of $\alpha+s_{\alpha}(\beta)$ considered as element of the dual $\mathfrak{t}^{*}$.

Proof. Let $\delta=s_{\alpha}(\beta)$. Then $\left(s_{\alpha} s_{\beta}(\lambda), \delta^{\vee}\right)<0$ hence $Y_{\delta} \notin \mathfrak{g}_{\left[v_{w(\lambda)}\right]}$ and $Y_{\delta} \notin \mathfrak{g}_{x}$.

In order to get: $Y_{\delta}+Y_{-\alpha} \in \mathfrak{g}_{x}$, we start to show

$$
\left(\lambda, \alpha^{\vee}\right)=\left(\lambda, \beta^{\vee}\right) \text {. }
$$

Set $\lambda=m \omega_{\alpha}+n \omega_{\beta}(m, n>0)$ and consider the variety $\mathrm{Cl}\left(T U_{\alpha} \cdot x\right)$. The arguments used in the proof of the previous corollary give a weight $\lambda_{i} \in \operatorname{supp} x$ such that $Y_{\alpha}^{r} \cdot v_{\lambda_{i}}$ is of weight $\lambda-\beta$ (for $r>0$ ). Translating the latter in terms of equations, we get $\lambda_{i}=\lambda-\alpha-n / m \delta$ and $n / m \in \mathbf{N}$. Similarly, considering the variety $\mathrm{Cl}\left(U_{\alpha} \cdot x\right)$, we get $m / n \in \mathbf{N}$. Therefore $m=n$ and

$$
Y_{-\alpha} \cdot v_{\lambda}=-q Y_{\delta} \cdot v_{\lambda_{i}}, \quad q \in \mathbb{C}^{*} .
$$


Finally, let $Z_{t}$ be the variety $\mathrm{Cl}\left(T \exp t\left(Y_{\delta}+q Y_{-\alpha}\right) \cdot x\right), t \in \mathbb{C}$. Its support is entirely contained in the triangle with vertices $\lambda, s_{\alpha}(\lambda)$ and $w(\lambda)$. But there is a gap in this support: the weight $\lambda-\alpha$ is missing because of (4). This implies that $\exp t\left(Y_{\delta}+q Y_{-\alpha}\right) \in G_{x}$.

To show that $U_{\gamma} \subset G_{x}$ for all $\gamma \in \Phi \backslash\{\alpha, \delta\}$, we proceed by contradiction and as before, we will find a gap in the support of $\mathrm{Cl}\left(U_{\gamma} \cdot x\right)$.

To conclude, we have to note that if $\langle\alpha, \beta\rangle$ is not of type $A_{1} \times A_{1}$, then $Y_{\alpha}+$ $Y_{-s_{\alpha}(\beta)} \notin \mathfrak{g}_{x}$. The first assertion is obtained just by symmetry.

As a consequence of the previous proof, we have

Corollary 6.5. (i) $\left(\lambda, \alpha^{\vee}\right)=\left(\lambda, \beta^{\vee}\right)$;

(ii) $T_{x}^{\circ}=\operatorname{ker}\left(\alpha+s_{\alpha}(\beta)\right)^{\circ}$;

(iii) $N_{G}(H) / H$ is finite;

(iv) The normalization of $\mathrm{Cl}(L \cdot x)$ is a cuspidal two-orbit L-variety.

The main thing to do now, in order to get the cuspidal two-orbit varieties of type II, is to give the list of the possible $(G, \alpha, \beta)$ where $\alpha$ and $\beta$ are the positive roots considered previously. If $G$ is of rank 2 , it is done already by Proposition 6.3. So the acting group $G$ will be definitely of rank greater than 2. Let us summarize the properties of $\alpha$ and $\beta$ :

1) $\alpha \in \Delta,(w(\lambda), \alpha) \leq 0$ and $U_{\alpha} \not \subset G_{x}$;

2) $\lambda-w(\lambda) \in\langle\alpha, \beta\rangle_{\mathbb{C}}$, the $\mathbb{C}$-vector space spanned by $\alpha$ and $\beta$;

3) $\{\alpha, \beta\}$ basis of $\langle\alpha, \beta\rangle$;

4) $\operatorname{supp} \beta \cup\{\alpha\}=\Delta$;

5) $w=s_{\alpha} s_{\beta}$;

6) $\left(\lambda, \alpha^{\vee}\right)=\left(\lambda, \beta^{\vee}\right)$.

It will appear quickly that there are very few roots satisfying all these conditions together mainly because of the two following statements.

Remark. Let $\gamma_{1}, \gamma_{2}$ and $\gamma_{3}$ be three roots of $\Phi$ spanning a root system $\Psi$ of rank 3. Suppose $\Psi$ satisfies the property:

$$
\eta=n_{1} \gamma_{1}+n_{2} \gamma_{2}+n_{3} \gamma_{3} \in \Psi \quad \Longrightarrow \quad \eta-n_{3} \gamma_{3} \in \Phi \quad \text { (up to a scalar). }
$$

If $\eta$ is a root of $\Phi$ then $\left\langle\gamma_{1}, \gamma_{2}\right\rangle_{\mathbb{C}} \cap\left\langle\gamma_{3}, \eta\right\rangle_{\mathbb{C}}$ is spanned by a root or is equal to $\{0\}$.

Consider a simple root $\delta$ such that $(\beta, \delta)>0($ then $\delta \in \operatorname{supp} \beta)$. Let $\gamma=s_{\alpha}(\delta)$. Then

$$
(w(\lambda), \gamma) \leq 0
$$

Lemma 6.6. If $\alpha, \beta$ and $\gamma$ satisfy the property given in the remark with $\gamma=\gamma_{3}$ then $(w(\lambda), \gamma)=0$. 
Proof. If $(w(\lambda), \gamma) \neq 0$ then according to $(5),(w(\lambda), \gamma)<0$. And therefore, there exists $\eta \in \Phi$ such that $\lambda-w(\lambda) \in\langle\gamma, \eta\rangle_{\mathbb{C}}$ (argue similarly as we did to get the root $\beta$ ). It implies: $\lambda-w(\lambda) \in\langle\alpha, \beta\rangle_{\mathbb{C}} \cap\langle\gamma, \eta\rangle_{\mathbb{C}}$. But this is impossible because of the remark and the fact that $T_{x}^{\circ}$ is regular (see Proposition 3.3).

Start with $G$ classical and suppose: $(\alpha, \delta)=0$. If $\operatorname{supp} \beta=\Delta$ then $\alpha, \beta$ and $\delta$ satisfy the conditions of Lemma 6.6. Therefore we get: $(\lambda, \delta)=(\lambda, \beta)$. But this equality is incompatible with $\left(\lambda, \alpha^{\vee}\right)=\left(\lambda, \beta^{\vee}\right)$. So assume that $\operatorname{supp} \beta=\Delta \backslash\{\alpha\}$. Then we have the following possibilities for $(G, \alpha, \beta)$ :

- $\left(A_{n}, \alpha_{i}(i=1, n), \tilde{\alpha}-\alpha_{i}\right)$;

- $\left(G, \alpha_{n}, \varepsilon_{1}-\varepsilon_{n}\right)$ for $G$ of type $B_{n}$ or $C_{n}$;

- $\left(G, \alpha_{1}, \varepsilon_{2}+\varepsilon_{n}\right)$ for $G$ of type $B_{n}$ or $C_{n}$;

- $\left(G, \alpha_{1}, \varepsilon_{2}+\varepsilon_{j}(2<j<n)\right)$ for $G$ of type $B_{n}, C_{n}$ or $D_{n}$;

- $\left(D_{n}, \alpha_{n-1}, \varepsilon_{1}+\varepsilon_{n}\right)$;

- $\left(D_{n}, \alpha_{n}, \varepsilon_{1}-\varepsilon_{n}\right)$.

Applying Lemma 6.6 case by case, we end up again with a contradiction. Thus necessarily, $(\alpha, \delta)<0$. The possible triples $(G, \alpha, \beta)$ are now:

- $\left(A_{3}, \alpha_{2}, \tilde{\alpha}\right)$;

- $\left(G, \alpha_{2}, \varepsilon_{1}+\varepsilon_{3}\right)$ for $G$ of type $B_{n}, C_{n}$ or $D_{n}$;

- $\left(B_{3}, \alpha_{3}, \tilde{\alpha}\right)$;

- $\left(B_{n}, \alpha_{1}, \varepsilon_{2}\right)$;

- $\left(C_{n}, \alpha_{2}, \tilde{\alpha}\right)$;

- $\left(C_{n}, \alpha_{1}, 2 \varepsilon_{2}\right)$;

- $\left(D_{n}, \alpha_{1}, \tilde{\alpha}\right)$.

Claim. In all these cases, $(w(\lambda), \gamma) \neq 0$.

But because of Lemma 6.6, we may also have $(w(\lambda), \gamma)=0$. So we have to reduce the list to

- $\left(A_{3}, \alpha_{2}, \tilde{\alpha}\right)$

- $\left(C_{3}, \alpha_{2}, \varepsilon_{1}+\varepsilon_{3}\right)$;

- $\left(B_{3}, \alpha_{3}, \tilde{\alpha}\right)$;

- $\left(C_{n}, \alpha_{2}, \tilde{\alpha}\right)$;

- $\left(D_{n}, \alpha_{1}, \tilde{\alpha}\right)$.

The fourth triple is ruled out just by considering $\left(w(\lambda), \alpha_{1}\right)$. The other ones give raise to some pairs of the table (apply Proposition 6.4).

For the exceptional case, we proceed similarly and we obtain the left pairs of the table. This ends the proof of Theorem 2.3. 


\section{References}

[1] D. Ahiezer, Dense orbits with two endpoints, Izv. Akad. Nauk SSSR Ser. Mat. 41 (1977), $\mathrm{n}^{\circ} 2,308-324$.

[2] D. Ahiezer, Equivariant completion of homogeneous algebraic varieties by homogeneous divisors, Ann. Global Anal. Geom. 1 (1983), 49-78.

[3] A. Bialynicki-Birula, On fixed point schemes of actions of multiplicative and additive groups, Topology 12 (1973), 99-103.

[4] A. Borel, Les bouts des espaces homogènes de groupes de Lie, Ann. of Math. 58 (1953), $443-457$.

[5] N. Bourbaki, Groupes et algèbres de Lie, Chapitres 4, 5 et 6, Masson, 1981.

[6] M. Brion, D. Luna and T. Vust, Espaces homogènes sphériques, Invent. Math. 84 (1986), $\mathrm{n}^{\circ} 3,617-632$.

[7] M. Brion, On spherical varieties of rank one, in: Group actions and invariant theory (Montreal, PQ, 1988), 31-41, CMS Conf. Proc. 10, Amer. Math. Soc., Providence, RI, 1989.

[8] M. Brion, A note on two-orbit varieties, in: Topology Hawaii (Honolulu, HI, 1990), 35-40, World Sci. Publishing, River Edge, NJ 1992.

[9] S. Cupit-Foutou, Classification des variétés à deux orbites, Prépublication de l'Institut de Recherche Mathématique Avancée, 2000/01, www-irma.u-strasbg.fr/irma/publications/2000/00001.shtml.

[10] D. Feldmüller, Two-orbit varieties with smaller orbit of codimension two, Arch. Math. (Basel) 54 (1990), $\mathrm{n}^{\circ}$ 6, 582-593.

[11] G. Horrocks, Fixed point scheme of additive group actions, Topology 8 (1969), 233-242

[12] A. Huckleberry and D. Snow, Almost-homogeneous Kähler manifolds with hypersurface orbits, Osaka J. Math. 19 (1982), no 4, 763-786.

[13] J. E. Humphreys, Linear algebraic groups, Springer, 1981.

[14] D. Luna and T. Vust, Plongements d'espaces homogènes, Comment. Math. Helv. 58 (1983), 186-245.

[15] D. Luna, Toute variété magnifique est sphérique, Transform. Groups 1 (1996), n 3, 249258.

[16] H. Sumihiro, Equivariant completion, J. Math. Kyoto Univ. 14 (1974), 1-28.

\section{S. Cupit-Foutou}

Bergische Universität

Gaussstr. 20

D-42097 Wuppertal

Deutschland

e-mail: cupit@math.uni-wuppertal.de

(Received: September 1, 2000) 